\title{
Electronic monitoring of adherence to inhaled corticosteroids: an essential tool in identifying severe asthma in children
}

\author{
Anja Jochmann ${ }^{1,2}$, Luca Artusio ${ }^{1}$, Angela Jamalzadeh¹, Prasad Nagakumar ${ }^{1}$, \\ Edgar Delgado-Eckert $\mathbb{1}^{2}$, Sejal Saglani ${ }^{1,3}$, Andrew Bush ${ }^{1,3}$, Urs Frey ${ }^{2}$ and \\ Louise J. Fleming ${ }^{1,3}$
}

Affiliations: ${ }^{1}$ Dept of Respiratory Paediatrics, Royal Brompton Hospital, London, UK. ${ }^{2}$ University of Basel, University Children's Hospital (UKBB), Basel, Switzerland. ${ }^{3}$ National Heart and Lung Institute, Imperial College London, London, UK.

Correspondence: Louise Fleming, Dept of Respiratory Paediatrics, Royal Brompton Hospital, Sydney Street, London, SW3 6NP, UK. E-mail: l.flemingarbht.nhs.uk

@ERSpublications

Children with true, severe therapy-resistant asthma cannot be identified without electronic adherence monitoring http://ow.ly/RMoU30fk1wu

Cite this article as: Jochmann A, Artusio L, Jamalzadeh A, et al. Electronic monitoring of adherence to inhaled corticosteroids: an essential tool in identifying severe asthma in children. Eur Respir J 2017; 50: 1700910 [https://doi.org/10.1183/13993003.00910-2017].

ABSTRACT International guidelines recommend that severe asthma can only be diagnosed after contributory factors, including adherence, have been addressed. Accurate assessment of adherence is difficult in clinical practice. We hypothesised that electronic monitoring in children would identify nonadherence, thus delineating the small number with true severe asthma.

Asthmatic children already prescribed inhaled corticosteroids were prospectively recruited and persistence of adherence assessed using electronic monitoring devices. Spirometry, airway inflammation and asthma control were measured at the start and end of the monitoring period.

93 children (62 male; median age 12.4 years) were monitored for a median of 92 days. Median (range) monitored adherence was $74 \%$ (21-99\%). We identified four groups: 1) good adherence during monitoring with improved control, 24\% (likely previous poor adherence); 2) good adherence with poor control, $18 \%$ (severe therapy-resistant asthma); 3) poor adherence with good control, 26\% (likely overtreated); and 4) poor adherence with poor control, $32 \%$. No clinical parameter prior to monitoring distinguished these groups.

Electronic monitoring is a useful tool for identifying children in whom a step up in treatment is indicated. Different approaches are needed in those who are controlled when adherent or who are nonadherent. Electronic monitoring is essential in a paediatric severe asthma clinic.

This article has supplementary material available from erj.ersjournals.com

Received: Nov 272016 | Accepted after revision: Sept 192017

Support statement: A. Jochmann received a Swiss National Science Foundation Early Post Doc Mobility Grant: P2SKP3_151971. Asthma UK Innovations Grant (AUK-IG-2014-284) to L.J. Fleming (principal investigator). A. Bush is a National Institute for Health Research (NIHR) senior investigator and principal investigator in the Asthma UK Centre for Applied Research. The project was supported by the NIHR Respiratory Disease Biomedical Research Unit at The Royal Brompton Hospital Foundation Trust and Imperial College London. S. Saglani is an NIHR Career Development Fellow. Funding information for this article has been deposited with the Crossref Funder Registry.

Conflict of interest: None declared.

Copyright OERS 2017 


\section{Introduction}

Suboptimal adherence to inhaled corticosteroids (ICSs) is the most common reason for treatment failure in asthma [1]. In children with apparently severe asthma referred to a tertiary centre, prescription pick-up was $<80 \%$ in over half the cohort [2]. Previous studies have demonstrated a link between suboptimal adherence and an increased risk of exacerbations, hospital admissions, asthma-related deaths and reduced lung function $[3,4]$.

Recent international guidelines for severe asthma recommend that nonadherence to treatment should be considered in all difficult to control patients. However, the authors acknowledge that detecting poor adherence can be challenging [5]. Physician assessment is little better than chance [4], and self- and parental-reported adherence overestimates treatment taken [6]. Prescription pick-up can act as a guide; those who pick up less prescriptions than they should are clearly not taking the intended dose. However, even when prescriptions are collected we do not know if the treatment is actually taken. Developing better tools for measuring adherence is important: 1) so that intervention strategies for adherence can be targeted to the appropriate patients [1], and 2) to avoid inappropriate investigations and escalation of treatment. Electronic monitoring devices can provide accurate data on medication usage, including timing and number of doses taken. However, previously these devices were not widely commercially available. Newer generations of electronic monitoring devices (e.g. Smartinhaler; Adherium, Auckland, New Zealand) can be used with a range of inhalers and the data downloaded remotely. This technology gives a clearer picture of adherence and how it impacts on asthma control and exacerbations. This information can potentially be used to guide asthma management.

In this study we monitored adherence using electronic monitoring devices in children with a range of asthma severities followed up in a tertiary asthma centre.

We hypothesised that electronic monitoring could separate patients with persistent severe symptoms despite proven good adherence from patients with poor adherence and potential for optimisation of standard asthma therapy, and that electronically monitoring adherence would lead to improvements in asthma control.

\section{Methods}

Study population and design

This was a prospective observational cohort study. Participants were recruited from the Outpatient Department, Royal Brompton Hospital, London, UK, between August 2014 and February 2015. All children were aged 5-17 years and had asthma diagnosed on the basis of evidence of one or more of the following: documented bronchodilator reversibility (BDR) $(\geqslant 12 \%)$, recorded evidence of spontaneous variation in forced expiratory volume in $1 \mathrm{~s}(\mathrm{FEV} 1)(\geqslant 12 \%)$ in the past year and airway hyperresponsiveness confirmed by direct or indirect challenge tests

Baseline visit: start of monitoring period

Asthma control (GINA, ACT/CACT)

Lung function, BDR

Indirect assessment of airway inflammation (FENO)

Asthma-related quality of life (mPAQLQ)

Smartinhaler issued

Adherence assessed (MARS-5, BMQ)

\begin{tabular}{|l|}
\hline Daily adherence \\
monitoring
\end{tabular}

FIGURE 1 Assessments at each study visit. GINA: Global Initiative for Asthma; ACT: Asthma Control Test; CACT: Childhood Asthma Control Test; BDR: bronchodilator reversibility; FENO: exhaled nitric oxide fraction; mPAQLQ: mini Paediatric Quality of Life Questionnaire; MARS-5: Medicines Adherence Rating Scale; BMQ: Beliefs about Medicines Questionnaire. 
The children recruited were classified into three groups based on treatment levels and previous assessments (see supplementary appendix A1). 1) Severe therapy-resistant asthma (STRA) group: children in whom potentially modifiable factors had previously been addressed and who continued to have either or both of ongoing poor control and acute asthma attacks despite high-dose ICSs plus add-on therapies (British Thoracic Society/Scottish Intercollegiate Guidelines Network guidelines stage 4/5 [7]). 2) Difficult asthma group: children previously or currently prescribed high-dose ICSs who had been found to have modifiable factors (such as poor adherence) as a cause for ongoing poor asthma control. 3) Mild-moderate asthma group: well or partly controlled according to Global Initiative for Asthma (GINA) guidelines [8] with a prescribed dose of ICSs of $\leqslant 250 \mu \mathrm{g}$ fluticasone propionate or $\leqslant 400 \mu \mathrm{g}$ budesonide propionate (or equivalent) per day with the need for none or no more than one controller medication (long-acting $\beta$-agonists, leukotriene receptor antagonists or theophylline).

Most children had previously been enrolled in our difficult asthma investigation protocol as described previously (see supplementary appendix A2) [2]. All children attended a baseline visit at the start of the monitoring period and follow-up visit 8-16 weeks later at the end of the monitoring period. Study visits were carried out as part of planned clinic visits, hence the variability in follow-up. Assessments carried out at the two study visits are shown in figure 1.

The original study was approved by the Regional Ethical Committee (NRES Committee London-Westminster). All parents/legal guardians gave written informed consent prior to any study procedures and the children gave age-appropriate assent or consent. The study was registered with ClinicalTrials.gov with identifier number NCT02252289.

\section{Study procedures \\ Adherence monitoring}

Inhaler technique competence was assessed by specialist asthma nurses in the clinic before the start of the study. Adherence was monitored using an electronic monitoring device (Smartinhaler). Smartinhalers were available for Symbicort, Seretide and Flixotide. One percent of devices had to be exchanged before being given out to a patient due to technical problems. In $5 \%$ of devices the battery died within a range of 2 weeks before the follow-up visit so this period had to be deducted from the adherence calculation. Participants and their guardians were informed that the Smartinhaler would record the total number of actuations of their ICS per day. It was attached to the participant's own inhaler in the clinic at the baseline visit. The devices contain a microchip that records the date and time each dose is taken. The data were downloaded at the follow-up visit by the study physician who managed the Smartinhaler set-up and software.

The Medicines Adherence Rating Scale (MARS-5) was used as a measure of self-reported adherence [9]. The Beliefs about Medicines Questionnaire (BMQ) was used to elucidate determinants of adherence [10].

Adherence was defined as the percentage of controller medication doses taken relative to the number of doses prescribed. Adherence was defined a priori as a ratio of doses taken (based on previously published definitions): good adherence $\geqslant 80 \%$ [11], moderate adherence $60-79 \%$ [12] and poor adherence $<60 \%$ [12].

The daily adherence was calculated with a maximum of $100 \%$, to avoid falsely increased values due to dose dumping.

\section{Assessments}

Asthma control was measured using the Asthma Control Test (ACT) for children aged $\geqslant 12$ years and the Childhood ACT (cACT) for children aged $<12$ years $[13,14]$.

Baseline spirometry to measure FEV1 and forced vital capacity (FVC) was performed using a spirometer (Vitalograph, Buckingham, UK) in accordance with American Thoracic Society (ATS)/European Respiratory Society (ERS) guidelines [15]. BDR testing was performed $15 \mathrm{~min}$ after administration of $1000 \mu \mathrm{g}$ salbutamol via a spacer.

Exhaled nitric oxide fraction (FENO) was measured using an online single-breath chemiluminescence analyser (NIOX VERO; Aerocrine, Stockholm, Sweden) at an expiratory flow rate of $50 \mathrm{~mL} \cdot \mathrm{s}^{-1}[16]$.

Quality of life and psychological morbidity were assessed using the mini Paediatric Asthma Quality of Life Questionnaire (mPAQLQ) [17].

Exacerbations in the 3 months prior to the baseline visit and during the monitoring period were recorded from the interview with the patients and the hospital records. Severe exacerbations were defined as $\geqslant 3$ days of oral prednisone prescription [18]. Moderate exacerbations were defined as the presence of one or more of deterioration in symptoms, deterioration in lung function and increased rescue bronchodilator use, lasting for $\geqslant 2$ days. Patients also noted their symptoms, any changes in medication and oral prednisolone courses in a symptom diary. 


\begin{tabular}{|c|c|c|}
\hline & Participants who completed the study & Dropouts \\
\hline Subjects & 93 & 15 \\
\hline Male & $62(67)$ & $9(60)$ \\
\hline Age years & $11.9 \pm 3.1$ & $14.1 \pm 3.0^{*}$ \\
\hline STRA & $21(23)$ & $2(13)$ \\
\hline Difficult asthma & 51 (55) & 8 (53) \\
\hline Mild-moderate asthma & $21(23)$ & 5 (33) \\
\hline Atopic & $82(88)$ & $13(87)$ \\
\hline Comorbidity & $17(18)$ & $8(53)^{*}$ \\
\hline \multicolumn{3}{|l|}{ Treatment } \\
\hline Median ICS dose (BDP equivalent) $\mu \mathrm{g} \cdot$ day $^{-1}$ & $800(100-3200)$ & $1000(200-2000)$ \\
\hline Leukotriene receptor antagonist & $55(59)$ & $8(53)$ \\
\hline Long-acting $\beta$-agonist & $91(98)$ & $14(93)$ \\
\hline Theophylline & $10(11)$ & $2(13)$ \\
\hline Maintenance OCS & $8(9)$ & $2(13)$ \\
\hline Omalizumab & $4(4)$ & $0(0)$ \\
\hline
\end{tabular}

Data are presented as $\mathrm{n}, \mathrm{n}(\%)$, mean \pm SD or median (range). STRA: severe therapy-resistant asthma; ICS: inhaled corticosteroid; BDP: budesonide propionate; OCS: oral corticosteroid. *: $p<0.05$.

Atopy was defined as one or more positive skin prick tests to a panel of common aeroallergens (mixed grasses, cat, dog, house dust mite, Aspergillus, trees, Alternaria, Cladosporium and Penicillium (wheal $\geqslant 3 \mathrm{~mm})$ ), or serum-specific IgE $\geqslant 0.34 \mathrm{kU} \cdot \mathrm{L}^{-1}$ in the last 3 years.

No prescribed treatment changes were made in any patient until the end of the monitoring period.

\section{Statistical analysis}

Statistical analysis was carried out using Prism (GraphPad, La Jolla, CA, USA) and R (www.r-project.org). Data were tested for normality using visual inspection, histograms and Kolmogorov-Smirnov testing. Parametric tests were used for normally distributed data. If the data were not normally distributed or there were only small numbers, nonparametric tests were used. The significance level was set at $\mathrm{p}<0.05$.

Differences over the monitoring period (for each of the parameters measured) were analysed using the Wilcoxon signed-rank or paired t-test.

Comparative ANOVA between two groups for continuous data was performed using the t-test for normally distributed data and the Mann-Whitney U-test for nonnormally distributed data, and for more than two groups the ANOVA or Kruskal-Wallis, respectively. Conservative Bonferroni correction was applied for multiple comparisons. A comparison of proportions between groups was performed using the Pearson Chi-squared test.

\section{Results}

A total of 108 children were recruited. 15 patients did not complete the study (supplementary figure S1). The data for the 93 children who completed the study are presented. Those who dropped out were significantly older and had more comorbidities (including migraine, focal epilepsy and delayed puberty) than those who completed the study. There were no other significant differences in baseline characteristics between the two groups (table 1).

\section{Adherence data}

The median (range) duration of monitoring was 92 (56-200) days. Median (range) adherence for the whole population was $74 \%$ (21-99\%). Good adherence was found in 39 patients $(42 \%)$ who took $\geqslant 80 \%$ of their prescribed medication, 25 patients (27\%) took $60-79 \%$ and 29 patients $(31 \%)$ took $<60 \%$ (supplementary table S2). Suboptimal adherence (adherence $<80 \%$ ) was seen in $58 \%$ of all patients in the study despite knowing they were being monitored.

There was no significant difference observed between the adherence groups with $\geqslant 80 \%, 60-79 \%$ or $<60 \%$ adherence in the following parameters at the start of monitoring: FENO, FVC, FEV1, BDR, ACT, cACT, mPAQLQ, exacerbation rate or oral corticosteroid courses. The proportion of STRA, difficult asthma or mild-moderate asthma patients was not significantly different between the adherence groups ( $\mathrm{p}=0.22$; data not shown). 
All patients and families stated that they took their asthma medication most of the time and as instructed by their physician, and the median MARS-5 score did not show any significant difference between the three adherence groups (24 versus 23 versus 23 for $\geqslant 80 \%, 60-79 \%$ or $<60 \%$ adherence, respectively; supplementary table S3).

The BMQ revealed a significantly higher score for the item relating to overuse and overprescription by healthcare professionals in the nonadherent group than in the adherent group (median BMQ score 2.2/5 versus 2/5) (supplementary table S3). There were no significant differences in the other domains (belief about necessity of medication, concern about medications or harm of medications). Prescription uptake data for the year prior to the start of the study were available for 51 patients (55\%). Prescription uptake and electronically monitored adherence showed no significant correlation. Electronically monitored adherence varied between $27 \%$ and $99 \%$ in participants with a prescription uptake of $100 \%$ (supplementary figure S2).

\section{Identification of clinically meaningful groups}

At the end of the monitoring period four groups could be identified according to their adherence and asthma control (ACT/cACT score). Clinical characteristics of these four groups are shown in table 2.

Group 1: previous poor control, but during monitoring good adherence and good control, $\mathrm{n}=22$ (24\%); these patients most likely improved their control by being adherent when they knew they were being monitored. Over the monitoring period there were significant improvements in FEV1, mPAQLQ and exacerbation rate after the Bonferroni correction for multiple testing.

Group 2: poor control of asthma before and during monitoring, despite good adherence, $\mathrm{n}=17$ (18\%); these children are likely to have true STRA requiring increased or add-on treatment. There were no significant improvements in this group.

Group 3: good asthma control $(\mathrm{ACT} / \mathrm{cACT} \geqslant 20)$ at the end of monitoring, despite suboptimal adherence in the monitoring period $(<80 \%), \mathrm{n}=24(26 \%)$; it is likely that these patients are overtreated. Over the monitoring period mPAQLQ improved significantly (after Bonferroni correction).

Group 4: suboptimal control and poor adherence, $n=30(32 \%)$; these patients need the reasons for poor adherence explored. There were no significant improvements in any measure of asthma control over the monitoring period.

Changes in asthma control over the monitoring period are displayed in table 3. All the tests were adjusted for conservative Bonferroni multiple comparison test due to having analysed a maximum of eight asthma control parameters in each category $(0.05 / 8$ interaction tests $=0.00625)$. These four groups and potential management options are shown in figure 2 .

Only the group with good adherence and good asthma control had a significant improvement across a range of asthma control measures at the end of the monitoring period. Those with good control at the end

TABLE 2 Baseline characteristics of four groups stratified according to adherence and asthma control at follow-up (Asthma Control Test (ACT) score)

\begin{tabular}{|c|c|c|c|c|}
\hline & \multicolumn{2}{|c|}{ Good adherence $(\geqslant 80 \%)$} & \multicolumn{2}{|c|}{ Suboptimal adherence $(<80 \%)$} \\
\hline & Group 1 (ACT $\geqslant 20)$ & Group 2 (ACT <20) & Group $3(A C T \geqslant 20)$ & Group $4(A C T<20)$ \\
\hline Patients & $22(24)$ & $17(18)$ & $24(26)$ & 30 (32) \\
\hline Age years & $11.5 \pm 2.8$ & $11.6 \pm 3.8$ & $12.2 \pm 2.5$ & $12.2 \pm 3.3$ \\
\hline Male & $16 / 22(73)$ & 10/17 (59) & $18 / 24(75)$ & $18 / 30(60)$ \\
\hline Caucasian & $13 / 22(59)$ & $12 / 17(71)$ & $10 / 24(42)$ & $16 / 30(53)$ \\
\hline Atopic & $18 / 22(82)$ & $16 / 17(94)$ & $20 / 24(83)$ & $28 / 30$ (93) \\
\hline BMI $\mathrm{kg} \cdot \mathrm{m}^{-2}$ & $18(14-29)$ & $20(14-30)$ & $21(15-39)$ & $20(14-32)$ \\
\hline Smoke exposure: yes & $6 / 22(27)$ & $3 / 17(18)$ & $3 / 24(13)$ & $6 / 30(20)$ \\
\hline FEV $1 \%$ pred & $88.3 \pm 15.4$ & $87.6 \pm 24.7$ & $83.7 \pm 19.2$ & $86.6 \pm 17.3$ \\
\hline BDR \% & $7.4(0-59)$ & $6.1(0-52)$ & $9.2(0-123)$ & $8.5(0-49)$ \\
\hline FENO ppb & $18(5-111)$ & $53(6-175)$ & $35.5(13-122)$ & $42.5(6-118)$ \\
\hline mPAQLQ score & $6.2(3-7)$ & $3.9(2-6.9)$ & $6.3(2.3-7)$ & $4.1(2.2-7.0)^{\#}$ \\
\hline
\end{tabular}

Data are presented as $\mathrm{n}(\%)$, mean $\pm \mathrm{SD}, \mathrm{n} / \mathrm{N}(\%)$ or median (range). BMI: body mass index; $\mathrm{FEV} 1$ : forced expiratory volume in $1 \mathrm{~s}$; BDR: bronchodilator reversibility; FENO: exhaled nitric oxide fraction; mPAQLQ: mini Paediatric Quality of Life Questionnaire. 
TABLE 3 Changes in asthma control over the monitoring period in the four groups stratified according to adherence and asthma control at follow-up (Asthma Control Test (ACT) score)

Visit

\begin{tabular}{lcc} 
& Visit & Good \\
\cline { 3 - 3 } & & Group 1 (ACT $\geqslant$ \\
Patients & & $22(24)$ \\
FEV $1 \%$ pred & Baseline & $88.3 \pm 15.4$ \\
& Follow-up & $98.9 \pm 16.4$ \\
& p-value & $0.002^{\#}$ \\
BDR \% & Baseline & $7.4(0-59)$ \\
& Follow-up & $2.3(0-12)$ \\
& p-value & 0.014 \\
FENo ppb & Baseline & $18(5-111)$ \\
& Follow-up & $11(5-85)$ \\
& p-value & 0.029 \\
mPAQLQ score & Baseline & $6.2(3-7)$ \\
& Follow-up & $6.8(5.5-7)$ \\
& p-value & $0.0003^{\#}$ \\
Exacerbations & Baseline & $1(0-10)$ \\
& Follow-up & $0(0-2)$ \\
& p-value & $0.005^{\#}$
\end{tabular}

Suboptimal adherence $(<80 \%)$

Group 3 (ACT $\geqslant 20) \quad$ Group 4 (ACT $<20)$

$\begin{array}{cc}24(26) & 30(32) \\ 83.7 \pm 19.2 & 86.6 \pm 17.3 \\ 89.9 \pm 13.4 & 91.0 \pm 15.5 \\ 0.134 & 0.060 \\ 9.2(0-123) & 8.5(0-49) \\ 3.8(0-42) & 4.5(0-20) \\ 0.023 & 0.044 \\ 35.5(13-122) & 42.5(6-118) \\ 33.0(9-96) & 31.5(8-193) \\ 0.015 & 0.419 \\ 6.3(2.3-7) & 4.1(2.2-7.0) \\ 6.7(4.8-7) & 4.6(2.6-6.7) \\ 0.006^{\#} & 0.074 \\ 1(0-10) & 1(0-8) \\ 0(0-2) & 1(0-4) \\ 0.039 & 0.891\end{array}$

Data are presented as $\mathrm{n}(\%)$, mean \pm SD or median (range), unless otherwise stated. FEV1: forced expiratory volume in $1 \mathrm{~s}$; BDR: bronchodilator reversibility; FENO: exhaled nitric oxide fraction; MPAQLQ: mini Paediatric Quality of Life Questionnaire. " significant after adjusting for Bonferroni multiple comparison test $(p<0.0065)$.

of monitoring despite suboptimal adherence also showed improvements across a range of measures, but only the increase in mPAQLQ reached statistical significance after Bonferroni correction. The improvement in FEV1 reached clinical significance according to the ATS definition. The ATS considers a minimally clinically significant difference (MCID) of $10 \%$ change in FEV1 relevant for adults [18]. There is little data available about the MCID in FEV 1 that children perceive. The ATS states that a change of $20 \%$ of FENO indicates a significant change following an intervention [19]. According to these recommendations a significant reduction was seen in the second group with good adherence and suboptimal control only. The MCID for ACT is 3 points, which was achieved in the group with good and moderate adherence (supplementary table S2) [20]. The MCID for the MPAQLQ is $\geqslant 0.5$ points [21], which was reached in all groups except the group with suboptimal adherence and good asthma control.

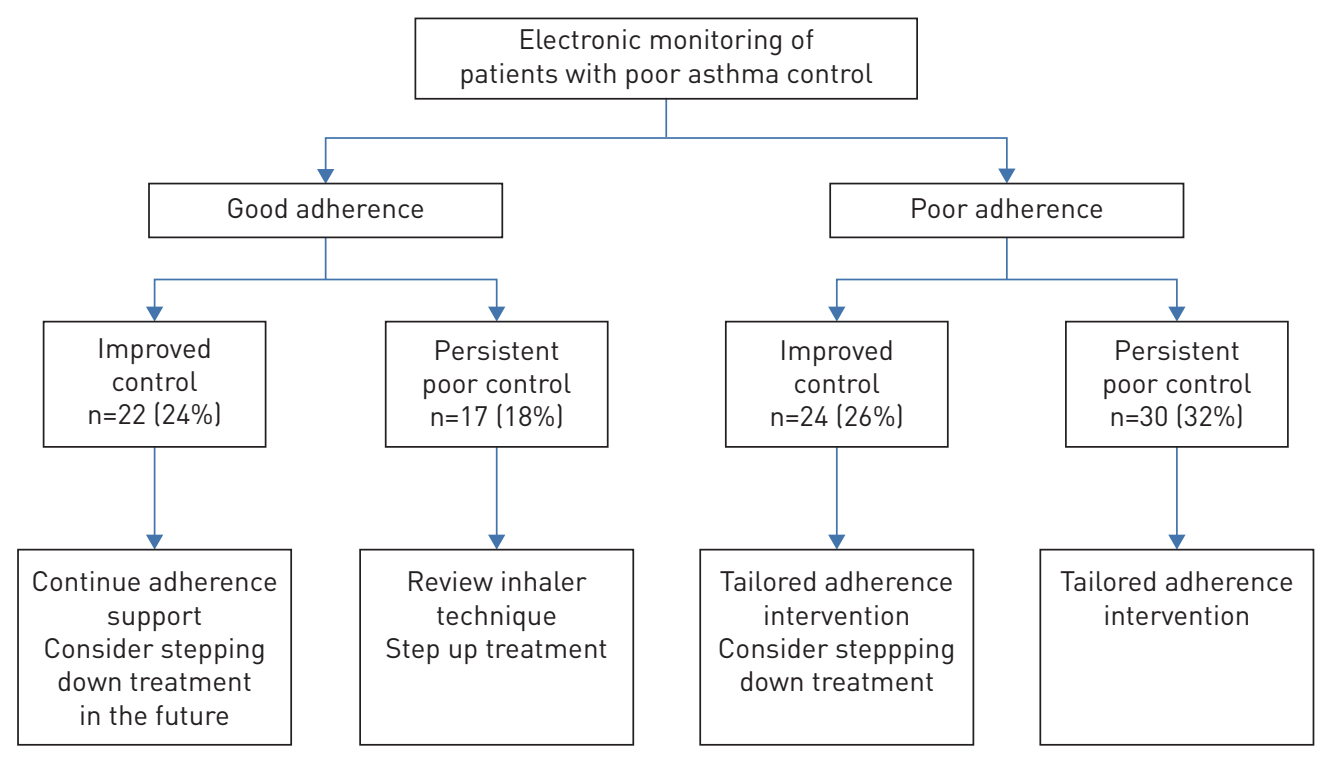

FIGURE 2 Management strategies based on asthma control and adherence during monitoring. 
Changes in asthma control over the monitoring period

FENO, FVC, FEV1, GINA control, ACT, cACT, mPAQLQ, number of exacerbations and BDR improved significantly over the monitoring period for the cohort as a whole (supplementary table S1). Those with adherence $\geqslant 80 \%$ improved in all asthma control parameters; those with adherence $60-79 \%$ improved in some, but not all asthma control parameters; those with adherence $<60 \%$ did not improve in any asthma control variable (supplementary table S2). There was a significant but weak correlation between adherence and FENO at the end of the monitoring period $(\mathrm{r}=0.46 ; \mathrm{p}<0.001)$. No correlation was found between adherence and any other parameter.

\section{Discussion}

This study has demonstrated the importance of accurate adherence monitoring in children with asthma. Suboptimal adherence $(<80 \%)$ was found in $58 \%$ of the children enrolled in this study despite knowing they were being monitored.

Asthma control improved following a period of monitoring in the cohort as a whole; however, this was not the case for all and, importantly, we were able to identify clinically meaningful subgroups. We have shown that this technique delineates two groups with good recorded adherence: a first group in whom symptoms, lung function and exacerbation rate improved, and who seemed to take more treatment after monitoring started as their control improved without any other intervention; and a second group who were adherent but whose control remained poor at the end of monitoring. Individuals in this latter group are likely to have true severe asthma and merit further phenotyping of their airway disease. We identified a nonadherent group, in whom the reasons for poor adherence need to be explored sensitively. Within this group some had improved control despite suboptimal adherence and were presumably being prescribed more treatment than they actually needed. Electronic monitoring only ensured good adherence in less than half the children in this study and therefore monitoring alone is not a sufficient intervention. However, we have demonstrated that electronic monitoring is an important tool to distinguish clinically important groups. Other factors that might have influenced improvement in asthma control cannot be excluded; however, the fact that the group with the worst adherence $(<60 \%)$ did not improve in any measure of asthma control highlights the importance of adherence monitoring. Furthermore, even those previously thought to have STRA who had undergone a detailed nurse-led assessment of adherence, including prescription uptake and a home visit as part of our difficult asthma protocol, were no more adherent than the other groups. Therefore, the distinction between difficult asthma and STRA cannot be made with certainty in the absence of electronic monitoring. We suggest that the gold standard definition for STRA must now include persistent poor control despite electronically recorded good adherence to treatment.

This study has again confirmed the inadequacy of self-reported adherence and the limitation of prescription uptake records. Using standard methods (exclusive of electronic monitoring), children with good adherence during the monitoring period were indistinguishable at baseline from those with poor adherence. This highlights the difficulties in detecting poorly adherent patients in clinical practice.

The improvements in asthma control over the monitoring period suggest that adherence improved over this time period as we made no changes in asthma treatment. We accept that we are not capturing the participant's "usual" adherence and that the electronic monitor is an intervention in itself even though it is not leading to improved adherence in all patients, but just in a subgroup. However, the information gained is of vital importance in determining future management, including tailoring adherence interventions. For those with suboptimal asthma control who were poorly adherent during monitoring it is unlikely that a monitoring-based system, even one that includes reminders, will be beneficial [22]. In this subgroup the individual patient barriers to adherence need to be explored and interventions tailored accordingly. Management needs to focus on the reasons for poor adherence; simply escalating the prescribed treatment will be futile. For those whose adherence improved due to monitoring, the challenge remains as to how to maintain good adherence beyond the monitoring period (or even during prolonged monitoring). Even in a comprehensive asthma care programme $41 \%$ of children did not manage to reach good adherence despite frequent consultations [3]. However, demonstrating that good control can be achieved may help to initiate a more sustained behaviour change. Further management in this group should focus on maintaining adherence and finding the optimal asthma treatment to maintain control.

Some of those with poor adherence had good control at the end of monitoring, suggesting that, although remaining suboptimal, their adherence had probably improved. Their prescribed asthma treatment could potentially be reduced. It is likely that treatment had previously been escalated because poor asthma control may have been attributed to undertreatment instead of nonadherence. Finally, those with ongoing poor control despite monitored good adherence are either manipulating the devices (either deliberately by dose dumping or because of poor technique) or are truly steroid resistant. The next step would be a period 
of directly observed therapy before administering expensive additional treatments such as omalizumab and other novel monoclonals.

We did not find a relationship between any of the measures of asthma control at baseline and monitored adherence. FENO at the end of the monitoring period had the best correlation with adherence compared with other parameters measured. The relationship between FENO and adherence was similar to the recently published data from KLOK et al. [23], who found FENO differed significantly at the end of monitoring between those with good and poor adherence. In those with poor control despite good adherence there was no significant reduction in FENO, again suggesting these are a truly steroid-resistant group or adherence was overestimated [24]. FENO is potentially a useful biomarker used in conjunction with adherence monitoring in determining management. In an exploratory analysis of participants with good adherence during electronic monitoring those whose FENO remained high were prescribed a higher dose of ICSs, and had worse lung function and asthma control than those who had a fall of at least $10 \mathrm{ppb}$ over the monitoring. This suggests that changes in FENO could be used to identify those with previously poor adherence whose adherence had improved because they were being monitored. As with the McNicholl et al. [25] study in adults, FENO suppression as a marker for improved adherence is most useful in those with high FENO at baseline. This is potentially a useful clinical tool for monitoring adherence, particularly as electronic monitoring devices are not widely available. However, $35 \%$ of our cohort had FENO levels $<25 \mathrm{ppb}$ at baseline and therefore FENO suppression cannot be used as a guide in these patients.

There are a number of limitations with the devices used in this study. The Smartinhaler records actuations and not whether the dose was actually inhaled or whether the inhaler was used correctly. Newer devices are in development which measure inhalational flow or sound. However, the differential improvements in those with good, moderate and poor adherence suggest that the monitored adherence was accurate. The costs of these devices is not insignificant (EUR100-150) and therefore funding was only available for the most common inhalers in our clinic (Symbicort, Seretide and Flixotide).

In this study $14 \%$ of participants did not complete the study and mislaid their Smartinhaler or failed to attend follow-up. Clearly no adherence information was available for these participants. It is possible that this is a reflection of a chaotic lifestyle which is likely to impact on adherence and therefore even a lost device can provide some useful information. Some may have felt that adherence monitoring was intrusive or unnecessary. Those who dropped out were older than those who completed the study and were more likely to have comorbidities. Adolescents, particularly those with other health needs, are a well-recognised risk group, and the importance of meaningful engagement with this group and ensuring they are not lost to follow-up during transition to adult services cannot be stressed strongly enough.

The cut-points for adherence categories were based on previous studies [11, 12]. The differential responses in asthma control between the three groups suggest that these are reasonable cut-points for defining good, moderate and poor adherence. However, we accept that other studies have used other definitions for good and poor adherence, and that these definitions are somewhat arbitrary. In some studies it has been shown that at least $80 \%$ of the prescribed dose needs to be taken to achieve optimal benefit [11]. In our study even those with lower adherence (60-79\%) showed some significant improvements (supplementary table S3). The lack of improvement in those with adherence $<60 \%$ suggests that this is a reasonable definition of poor or nonadherence and is in keeping with the study by McNALLY et al. [12], which found a significant difference in healthcare utilisation in those with adherence $<62 \%$.

The monitoring period in this study was relatively short and the impact of monitoring as an intervention is likely to wane with time [26]. Furthermore, we acknowledge that this was not an interventional study as there was no control group and therefore improvement cannot be attributed solely to adherence monitoring. Unmeasured influences or regression to the mean might also have an effect.

Although one would hope that the demonstration of improved control with good adherence would help to initiate behaviour change in the individual, this is perhaps an overoptimistic view and the design of this study does not enable us to answer that question. Electronic monitoring studies that have used feedback and reminders have shown impressive differences in adherence in the intervention group, but more modest improvements in asthma control [26, 27]. This may be because the monitoring devices used measured actuations and not inhalation, and participants in the intervention arm may have been manipulating the devices or had poor technique. Furthermore, interventions for those whose adherence remains poor when monitored are likely to be different to those who do well during a period of monitoring. For example, using reminders will help those who frequently forget [28], but will have little impact on those who are concerned about side-effects or think the inhalers are ineffective and for whom an educational intervention would be more appropriate [29-31]. 
Further research is needed to assess the utility of combining electronic monitoring devices and tailored adherence interventions. However, this study has clearly demonstrated that electronic monitoring is essential if poor adherence is to be excluded as a cause of poor asthma control. Furthermore, the technique can be used to identify clinically meaningful subgroups whose ongoing management could be determined by a combination of adherence assessment and clinical control. We conclude that it is not possible to optimally manage severe asthma without the availability of objective monitoring of adherence.

\section{Acknowledgements}

We would like to thank all the children and their families who participated in the study. We are grateful to Claire Hogg, Mark Rosenthal and Ian Balfour-Lynn (Royal Brompton Hospital, London, UK) for allowing recruitment of their patients, and Winston Banya (Royal Brompton Hospital, London, UK) for additional statistical advice.

Author contributions: A. Jochmann contributed to the conception and design of the work, the acquisition, analysis and interpretation of data as well as drafting the manuscript and revising it. L. Artusio, A. Jamalzadeh and P. Nagakumar contributed to the acquisition and interpretation of data. E. Delgado-Eckert contributed to analysing the data. S. Saglani, A. Bush and U. Frey contributed to data interpretation and revising the work critically. L. Fleming contributed to the conception and design of the work, the interpretation of data as well as drafting the manuscript and revising it. All authors approved the final version of the manuscript to be published.

\section{References}

1 Bender B, Milgrom H, Apter A. Adherence intervention research: what have we learned and what do we do next? J Allergy Clin Immunol 2003; 112: 489-494.

2 Bracken M, Fleming L, Hall P, et al. The importance of nurse-led home visits in the assessment of children with problematic asthma. Arch Dis Child 2009; 94: 780-784.

3 Klok T, Kaptein AA, Duiverman EJ, et al. Long-term adherence to inhaled corticosteroids in children with asthma: observational study. Respir Med 2015; 109: 1114-1119.

4 Burgess SW, Sly PD, Morawska A, et al. Assessing adherence and factors associated with adherence in young children with asthma. Respirology 2008; 13: 559-563.

5 Chung KF, Wenzel SE, Brozek JL, et al. International ERS/ATS guidelines on definition, evaluation and treatment of severe asthma. Eur Respir J 2014; 43: 343-373.

6 Bender B, Wamboldt FS, O'Connor SL, et al. Measurement of children's asthma medication adherence by self report, mother report, canister weight, and Doser CT. Ann Allergy Asthma Immunol 2000; 85: 416-421.

7 British Thoracic Society/Scottish Intercollegiate Guidelines Network. BTS/SIGN Asthma Guideline. 2014. https:// www.brit-thoracic.org.uk/guidelines-and-quality-standards/asthma-guideline/2014 Date last accessed: January 31, 2017.

8 Global Initiative for Asthma. Pocket Guide for Asthma Management and Prevention. 2016. http://ginasthma.org/ wp-content/uploads/2016/05/WMS-GINA-2016-main-Pocket-Guide.pdf Date last accessed: October 31, 2016.

9 Horne R, Weinman J. Self-regulation and self-management in asthma: exploring the role of illness perceptions and treatment beliefs in explaining non-adherence to preventer medication. Psychol Health 2002; 17: 17-32.

10 Horne R, Weinman J, Hankins M. The beliefs about medicines questionnaire: the development and evaluation of a new method for assessing the cognitive representation of medication. Psychol Health 1999; 14: 1-24.

11 Santos PdeM, D'Oliveira A Jr, Noblat LdeA, et al. Predictors of adherence to treatment in patients with severe asthma treated at a referral center in Bahia, Brazil. J Bras Pneumol 2008; 34: 995-1002.

12 McNally KA, Rohan J, Schluchter M, et al. Adherence to combined montelukast and fluticasone treatment in economically disadvantaged African American youth with asthma. J Asthma 2009; 46: 921-927.

13 Nathan RA, Sorkness CA, Kosinski M, et al. Development of the Asthma Control Test: a survey for assessing asthma control. J Allergy Clin Immunol 2004; 113: 59-65.

14 Liu AH, Zeiger R, Sorkness C, et al. Development and cross-sectional validation of the Childhood Asthma Control Test. J Allergy Clin Immunol 2007; 119: 817-825.

15 Miller MR, Hankinson J, Brusasco V, et al. Standardisation of spirometry. Eur Respir J 2005; 26: 319-338.

16 American Thoracic Society, European Respiratory Society. ATS/ERS recommendations for standardized procedures for the online and offline measurement of exhaled lower respiratory nitric oxide and nasal nitric oxide, 2005. Am J Respir Crit Care Med 2005; 171: 912-930.

17 Juniper EF, Guyatt GH, Feeny DH, et al. Measuring quality of life in children with asthma. Qual Life Res 1996; 5: 35-46.

18 Reddel HK, Taylor DR, Bateman ED, et al. An official American Thoracic Society/European Respiratory Society statement: asthma control and exacerbations: standardizing endpoints for clinical asthma trials and clinical practice. Am J Respir Crit Care Med 2009; 180: 59-99.

19 Dweik RA, Boggs PB, Erzurum SC, et al. An official ATS clinical practice guideline: interpretation of exhaled nitric oxide levels (FENO) for clinical applications. Am J Respir Crit Care Med 2011; 184: 602-615.

20 Dinakar C, Chipps BE. Clinical tools to assess asthma control in children. Pediatrics 2017; 139: e20163438.

21 van Bragt S, van den Bemt L, Kievits R, et al. PELICAN: a cluster-randomized controlled trial in Dutch general practices to assess a self-management support intervention based on individual goals for children with asthma. $J$ Asthma 2015; 52: 211-219.

22 Vasbinder EC, Goossens LM, Rutten-van Molken MP, et al. e-Monitoring of Asthma Therapy to Improve Compliance in children (e-MATIC): a randomised controlled trial. Eur Respir J 2016; 48: 758-767.

23 Klok T, Brand PL. Can exhaled nitric oxide fraction predict adherence to inhaled corticosteroids in atopic and nonatopic children with asthma? J Allergy Clin Immunol Pract 2017; 5: 521-522.

24 Sulaiman I, Seheult J, MacHale E, et al. A method to calculate adherence to inhaled therapy that reflects the changes in clinical features of asthma. Ann Am Thorac Soc 2016; 13: 1894-1903.

25 McNicholl DM, Stevenson M, McGarvey LP, et al. The utility of fractional exhaled nitric oxide suppression in the identification of nonadherence in difficult asthma. Am J Respir Crit Care Med 2012; 186: 1102-1108. 
26 Morton RW, Elphick HE, Rigby AS, et al. STAAR: a randomised controlled trial of electronic adherence monitoring with reminder alarms and feedback to improve clinical outcomes for children with asthma. Thorax 2017; 72: 347-354.

27 Chan AH, Stewart AW, Harrison J, et al. The effect of an electronic monitoring device with audiovisual reminder function on adherence to inhaled corticosteroids and school attendance in children with asthma: a randomised controlled trial. Lancet Respir Med 2015; 3: 210-219.

28 Foster JM, Smith L, Usherwood T, et al. The reliability and patient acceptability of the SmartTrack device: a new electronic monitor and reminder device for metered dose inhalers. J Asthma 2012; 49: 657-662.

29 Marcus BH, Goldstein MG, Jette A, et al. Training physicians to conduct physical activity counseling. Prev Med 1997; 26: 382-388.

30 Butler CC, Rollnick S, Cohen D, et al. Motivational consulting versus brief advice for smokers in general practice: a randomized trial. Br J Gen Pract 1999; 49: 611-616.

31 DiIorio C, McCarty F, Resnicow K, et al. Using motivational interviewing to promote adherence to antiretroviral medications: a randomized controlled study. AIDS Care 2008; 20: 273-283. 